\title{
Téoros
}

Revue de recherche en tourisme

\section{Le Québec : destination internationale}

\section{Marie-Hélène Courtade et Guy Leroux}

Volume 6, numéro 3, décembre 1987

Le tourisme, phénomène mondial

URI : https://id.erudit.org/iderudit/1080604ar

DOI : https://doi.org/10.7202/1080604ar

Aller au sommaire du numéro

Éditeur(s)

Université du Québec à Montréal

ISSN

0712-8657 (imprimé)

1923-2705 (numérique)

Découvrir la revue

Citer cet article

Courtade, M.-H. \& Leroux, G. (1987). Le Québec : destination internationale. Téoros, 6(3), 33-36. https://doi.org/10.7202/1080604ar d'utilisation que vous pouvez consulter en ligne.

https://apropos.erudit.org/fr/usagers/politique-dutilisation/ 

Marie-Hélène Courtade
et Guy Leroux*

\section{Le Québec: destination internationale}

Quelle est la proportion relative des voyageurs des autres pays entrant au Québec par rapport à l'ensemble des voyageurs internationaux au Canada et en Ontario? D'où viennent-ils? Quelles sont les tendances constatées au cours des dix dernières années? Ce seront les questions auxquelles nous tenterons d'abord de répondre pour quantifier le tourisme international au Québec. De plus, étant donné l'importance des voyageurs américains comme touristes étrangers au Canada et au Québec, on s'attardera à un aspect plus qualitatif, en discutant des perceptions de notre produit touristique par les américains qui ont l'intention de faire un voyage d'agrément au Québec.

Pour quantifier le tourisme international au pays et dans chacune des provinces, les seules donnees récurrentes sont recueillies par Statistique Canada et regroupées dans la publication "Voyages entre le Canada et les autres pays" (66-001 au catalogue). La direction de l'analyse et du développement du ministère du Tourisme du Québec effectue, de façon mensuelle, un traitement de diverses statistiques relatives au tourisme international qu'elle publie sous la forme d'un "Baromètre touristique du Québec" et dans lequel on retrouve un certain nombre d'indicateurs qui permettent de cerner, de façon plus adéquate, cette industrie au Québec.

Il est important de préciser ici que le nombre de touristes entrés au pays est calculé à partir des "entrées aux frontières". Tel que précisé dans le Baromètre touristique: ". ... Les entrées aux frontières sont un indicateur partiel du véritable nombre de résidants des Etats-Unis ou des autres pays venus dans ces provinces. Elles $n$ 'indiquent pas le nombre de résidants américains ou des autres pays que des Etats-Unis venus via les portes d'entrées des autres provinces".

\section{7-1987: dix années tourmentées}

Après une croissance importante et constante des entrées de voyageurs internationaux au Canada entre 1977 et 1981 , on note une certaine hésitation dans la courbe évolutive récente. En effet, alors que les entreses totales de voyageurs étrangers au Canada se situaient à un niveau légèrement supérieur à 33 millions en 1977, une augmentation annuelle moyenne de $6,6 \%$ portait ce nombre à près de 42 millions en 1981 (graphique 1). Après une chute importante de $23 \%$ l'année suivante, la tendance dans les entrées de voyageurs internationaux au pays accuse, à nouveau, une croissance continue pour atteindre, en 1986 , près de 40,5 millions. Si l'on compare les résultats du premier trimestre de 1987 à ceux de 1986 pour la même période, on constate une augmentation de $3,9 \%$. Si la tendance se poursuit pour les trois autres trimestres de 1987, il est probable que le sommet des entrées de voyageurs internationaux au Canada, atteint en 1981, sera enfin dépassé.

Il n'est pas surprenant de constater que la très grande majorité des visiteurs étrangers au Canada soient des résidents des États-Unis. Pour l'année 1986, ils représentent 94,4\% des entrées totales, soit plus de 38 millions de voyageurs. Malgré tout, il est quand mềne intéressant d'examiner la répartition et l'ćvolution des principaux segments de clientèles étrangères. Sur plus de 2,25 millions de visiteurs étrangers autres que des États-Unis, les deux plus importantes régions émettrices de touristes vers le Canada sont l'Europe et l'Asie avec, respectivement, $57,7 \%$ et $25 \%$ de ce marché en 1986 (graphique 2). De plus, depuis 1977, deux régions présentent un taux de croissance particulièrement intéressant pour le pays. En effet, l'Asie et l'Océanie ont vu leur nombre de touristes à destination du Canada s'accroître, respectivement, de $158,5 \%$ et $80,3 \%$ entre 1977 et 1986 .

Il est acquis que l'industrie touristique a un impact non négligeable sur l'économie du pays. Pour 1986, le niveau des recettes provenant de l'ensemble des touristes étrangers atteignait près de 6,4 milliards de dollars. Par contre, les touristes canadiens dépensaient, quant à eux, près de 7,5 milliards à l'extérieur du pays, portant le déficit au compte des voyages internationaux à un peu plus d'un milliard de dollars (graphique 3). En effet, depuis vingt ans, c'est seulement en
1967, année de l'Exposition internationale de Montréal, que le Canada a connu une balance positive au compte des voyages internationaux. Bien qu’à cet égard le Canada ait enregistré une balance positive avec les Etats-Unis entre 1966 et 1974, l'effet combiné par rapport aux autres pays a constamment placé le Canada dans une situation déficitaire. Mais, si la balance au compte des voyages internationaux avec les États-Unis s'est révélée également déficitaire entre 1975 et 1985 , la situation s'est constamment améliorée depuis 1983 pour atteindre un niveau positif, pour le Canada, l'an dernier.

\section{Le Québec, à l'image du tourisme canadien}

Au cours des dix dernières années, le Québec a connu une situation comparable à celle observée à l'échelle du pays. En effet, le nombre de voyageurs non-résidents entrés au Canada via le Québec atteignait en 1980 son plus haut niveau, soit près de 3,6 millions d'entrées. Ce sommet était presque à nouveau atteint en 1986 alors qu'on enregistrait 3,4 millions d'entrées de touristes étrangers à nos frontières. En fait, il est intéressant de constater qu'entre 1977 et 1986 le nombre de touristes étrangers au Québec a augmenté globalement de $2,5 \%$ et que cette augmentation est principalement due à la croissance des marchés étrangers, autres qu'américains, qui ont enregistré pour la mème période une augmentation de $31 \%$.

Malgré tout, l'importance quantitative des touristes étrangers autres qu'Américains dans la province demeure relativement faible. En 1986 , la proportion relative de ce segment sur l'ensemble des touristes étrangers était de $10,1 \%$ sur près de 3,5 milions de visiteurs étrangers. Tout comme pour l'ensemble du pays, les Américains, avec plus de 3 millions d'entrées enregistrées à nos frontières pour la même année, constituent l'essentiel de notre clientèle touristique. Le marché international autre qu'américain représente, quant à lui, un peu moins de 400000 "visitesprovinces".

A nouveau, la répartition des voyageurs résidents des pays autres que les Etats-Unis entrés au Québec est comparable à la situation qui prévaut à l'échelle nationale. Ainsi, pour 1986 , près de $70 \%$ de ces voyageurs originaient de l'Europe, $12 \%$ étaient originaires d'Asie et $7 \%$ provenaient d'un pays de l'Amérique du nord (autre que les ÉtatsUnis), de l'Amérique centrale et des Antilles (graphique 4). En Europe, les trois pays 
émetteurs du plus grand nombre de voyageurs au Québec sont, dans l'ordre, la France, le Royaume Uni et la République Fédérale d'Allemagne avec, respectivement, $36,1 \%, 13,9 \%$ et $8,8 \%$ de ce marché en 1986. L'Italie, la Suisse et la Pologne suivent de près avec, environ $6 \%$ du marché chacun. Quant aux régions de l'Asie, ce sont Israel et le Japon qui représentent les plus forts segments de ce marché alors que le Mexique et Haiti sont les régions d'Amérique du Nord les plus fortement émettrices de voyageurs vers le Québec.

À cette étape-ci, il est intéressant de situer le Québec par rapport au Canada et de comparer ses performances à celles de l'Ontario. Ainsi, le nombre de voyageurs étrangers entrés au Canada via le Québec et via l'Ontario représentait, en 1977 et en 1986, plus de $70 \%$ des entrées totales au Canada. De plus, sur le nombre d'entrées au Canada via le Québec et l'Ontario, on constate que notre part de marché est plus forte pour les voyageurs des pays autres que les Etats-Unis que pour les voyageurs américains soit, respectivement $24 \%$ et $11 \%$ pour l'année 1986 (graphique 5).

\section{Comment les Américains perçoivent-ils le Québec?}

De toute évidence, la clientèle américaine constitue la principale clientèle touristique internationale du Canada et, plus particulièrement, du Québec, tant pour les' voyages d'affaires que pour les voyages d'agrément. Même si le nombre de touristes internationaux non-américains tend à augmenter, notre proximité géographique du marché américain favorise pour cette population, la visite de la province. Toutefois, les particularités du Québec qui devraient intéresser nos voisins du sud n'ont pas tous été exploités. De plus, la méconnaissance du comportement de voyage des Américains, en termes qualitatifs, de même que les similarités entre les produits touristiques americain et canadien sont des facteurs qui rendent difficile la segmentation d'un marché à fort potentiel.

Pour pallier, entre autres, au manque d'informations qualitatives sur le marché américain des woyages d'agrément, Tourisme Canada faisait réaliser une vaste enquête en 1985 , auprès de 9000 américains répartis dans les neuf (9) divisions de recensement des EtatsUnis, soient environ 1,000 répondants par division. Cetté étude, réalisée à partir d'entrevue personnelle, visait à donner un portrait détaillé des voyages passés et des projets de voyages des Américains, à connaître leur comportement de voyage et. plus particulièrement, leurs perceptions quant au produit touristique canadien. Les perceptions décrites ici sont celles des Américains qui projetaient, de façon certaine et probable, d'effectuer un voyage d'agrément au Québec au cours des deux années suivant l'entrevue. Pour cette enquête, le marché américain des voyages d'agrément a êté segmenté en huit types de voyages distincts pour lesquels des ensembles de perceptions différentes ont été identifiés.

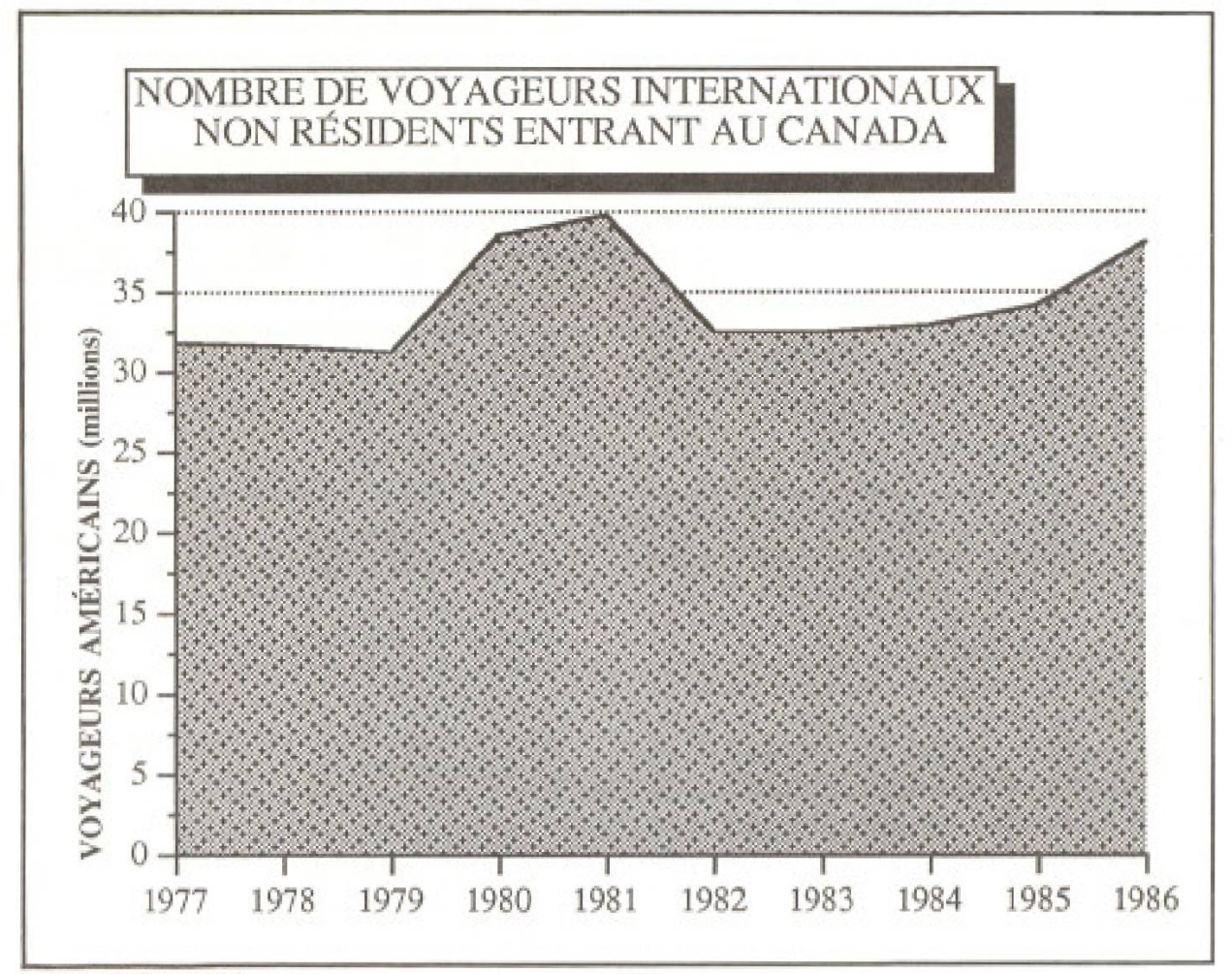

GRAPHIQUE 1

GRAPHIQUE 2

REPARTITION DES VOYAGEURS INTERNATIONAUX, AUTRES QUE DES ETTATS-UNIS, AU CANADA

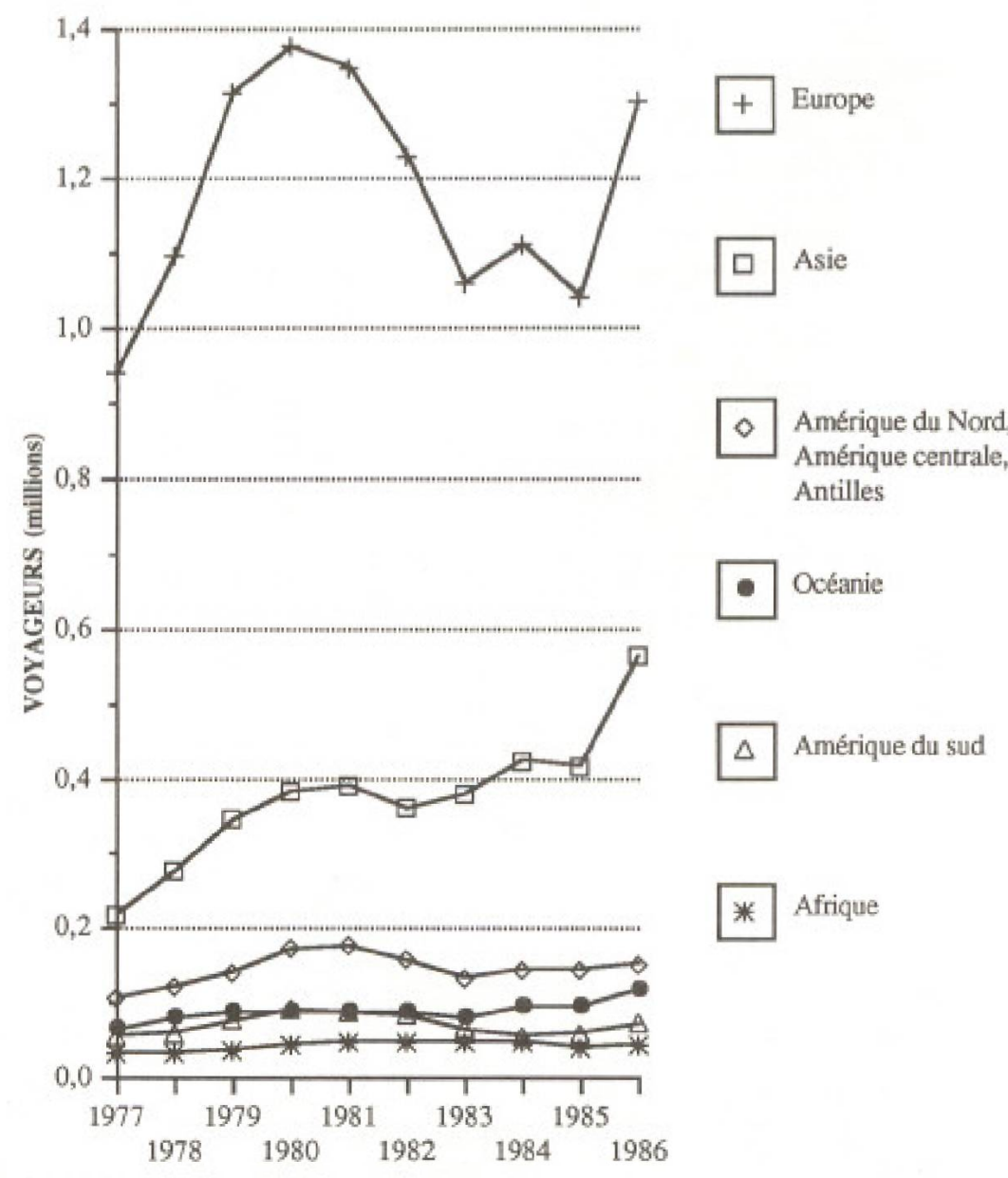


La première grande conclusion de cette étude a fait ressortir que les deux types de voyages au Québec les plus fréquemment projetés par les Americains étaient les voyages dans une grande ville et les circuits touristiques; ils représentaient respectivement $37 \%$ et $27 \%$ de l'ensemble des voyages projetés dans la province par les Américains.

Dans le premier cas, le Québec, par sa notoriété, son infrastructure de qualité et ses nombreuses activités, était perçu par les Américains comme une destination privilégiếe pour les séjours dans une grande ville. Les Américains ayant l'intention d'y effectuer un voyage d'agrément espèrent y trouver ce qu'ils recherchent généralement dans le cadre de ce type de voyage, soit:

- des villes célèbres et populaires;

- des hôtels de première classe;

- des lieux où on ne risque pas de s'ennuyer;

- des monuments et des points d'intérêt:

- des restaurants élégants.

En fait, c'est surtout par rapport aux autres villes canadiennes que Montréal et Québec bénéficient d'avantages distinctifs tels que la popularité, la différence culturelle, l'aspect sécuritaire et la propreté. Par contre, les deux grandes villes touristiques de la province sont moins bien perçues quant aux prix, étant évaluées comme des destinations coutteuses. L'intérêt des voyages dans une grande ville est d'autant moins négligeable que ce type de voyage constitue bien souvent la porte d'entrée que prennent les voyageurs américains vers d'autres régions touristiques du Québec; ils revêtent donc une importance fort significative pour l'ensemble du tourisme québecois.

A l'instar des voyages dans une grande ville, le Québec est perçu comme possédant les atouts qui en font une destination touristique par excellence dans le cadre d'un voyage de type circuit, soit:

- la beauté du paysage:

- le nombre de choses à voir et à faire et donc, le faible risque de s'ennuyer;

- des endroits bien connus et populaires;

- des monuments et des points d'intérêt bien connus.

De nouveau, le Québec est une destination de choix par ses aspects nouveaux et différents en matière de culture et de mode de vie. Le Québec est également considéré comme une région dotée d'une infrastructure hơtelière et de restauration de première classe et comme un territoire ou le voyage est sécuritaire. Par contre, la province est de nouveau perçue comme une destination coûteuse, principale ombre dans l'image perceptuelle de la destination sur le marché américain des voyages d'agrément.

Enfin, les deux autres types de voyage d'agrément qu'on associe, de façon beaucoup moins prononcée, au Québee sont les voyages de plein air et les voyages dans un centre de villegiature.

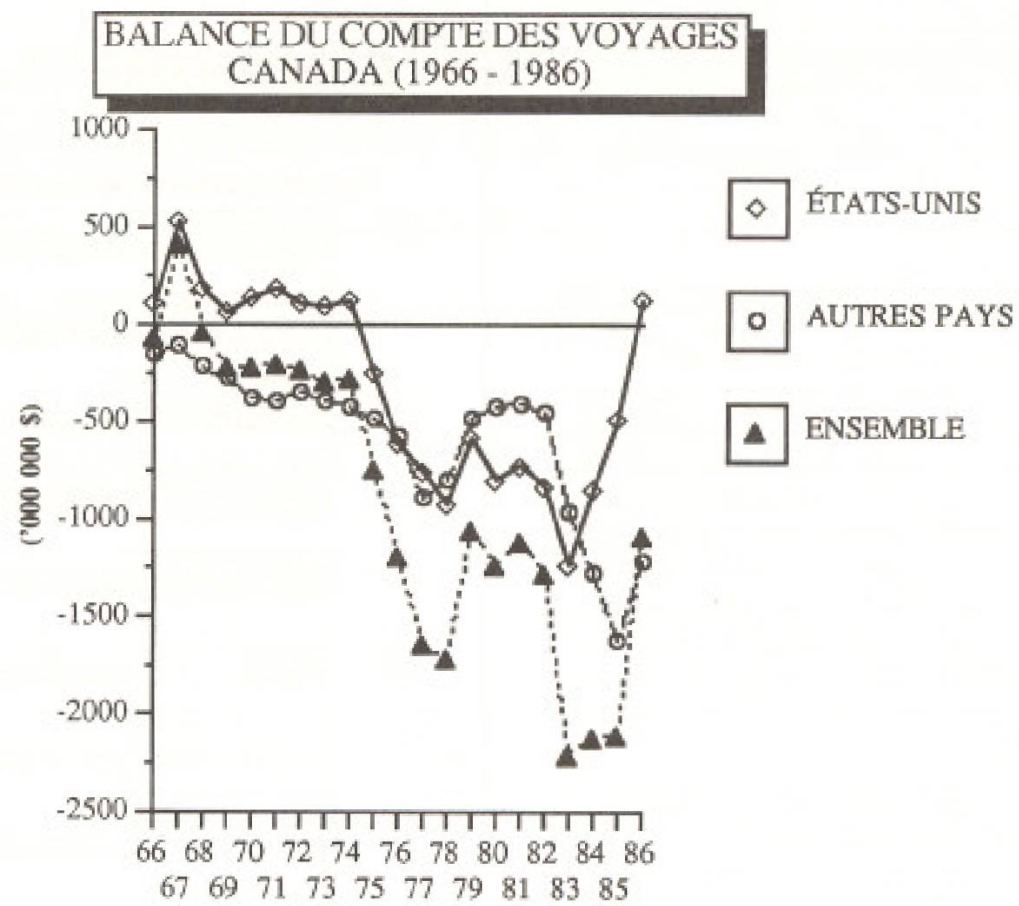

ANNÉE

GRAPHIQUE 3

GRAPHIQUE 4

REPARTITION DES VOYAGEURS INTERNATIONAUX, AUTRES QUE DES ETATS-UNIS, AU QUÉBEC

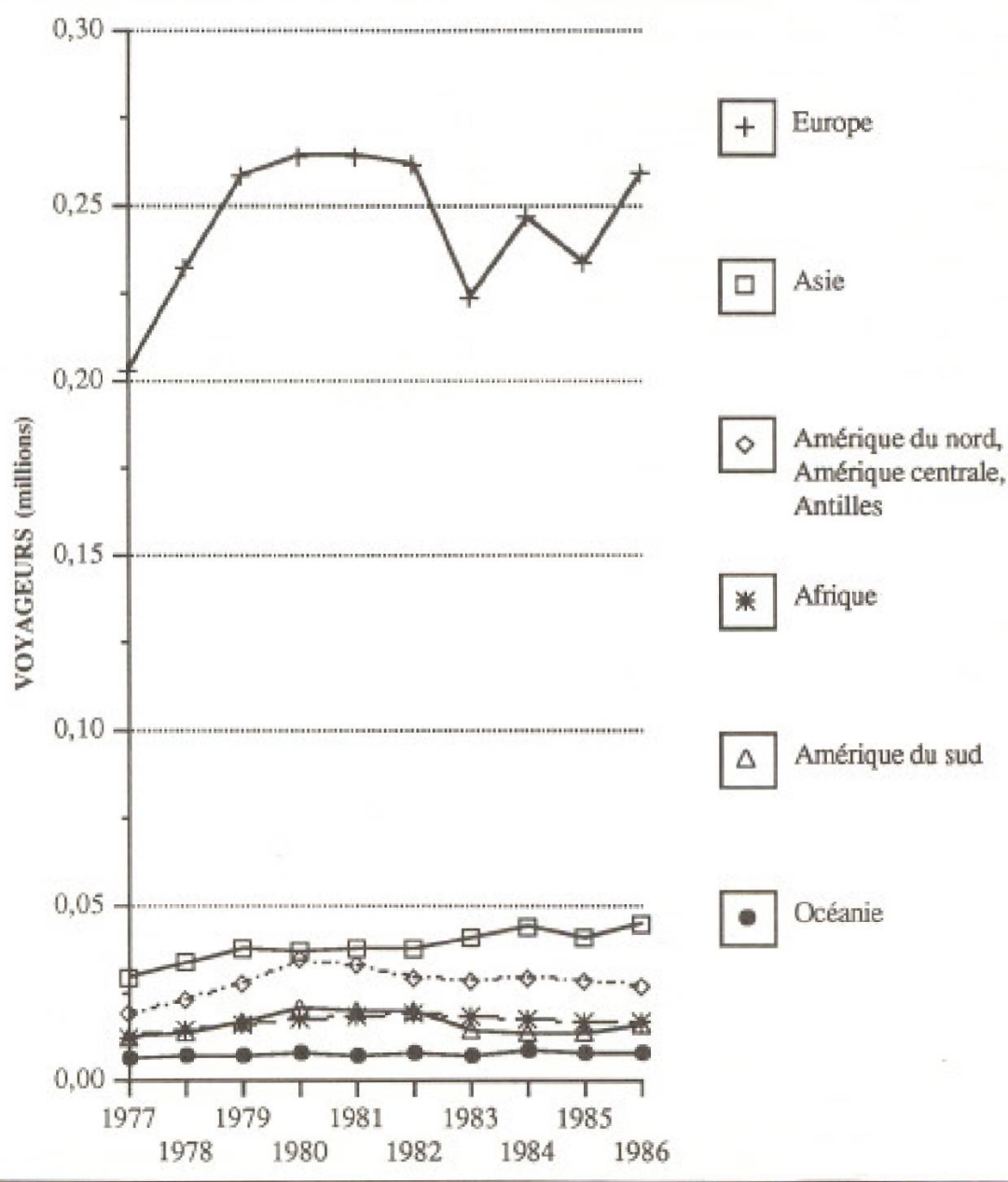

35 TEOROS - Val. 4, no 3, decentare 1967 
Ainsi, dans le cas des voyages de plein air, on reconnait au Québec certaines des caractéristiques recherchées par les adeptes de ce type de voyage, notamment:

- la beauté du paysage;

- beaucoup de choses à voir et à faire;

- des régions sauvages mais accessibles.

- une impression d'aventure.

De plus, les Américains attribuent au Québec des avantages distinctifs par rapport aे l'ensemble des destinations canadiennes de plein air. Pour eux, la province se distingue, à nouveau, par sa difference culturelle et par son intếrêt historique. Néanmoins, ce ne sont pas ces caractéristiques perceptuelles qui font d'une destination une région de plein air privilégiée. A cet égard, le Québec est nettement désayantagé par sa situation géographique: les voyages de plein air étant bien souvent de courte durbe, on cherche a les faire dans une région relativement près de chez soil.

Dans le même ordre d'idée, le Québec ne répond pas aux critères recherchés lors du choix d'une destination de type centre de villégiature. Dans ce cas-ci, les Américains recherchent avant tout du soleil, la mer, la possibilité de pratiquer des sports nautiques tout en se relaxant.

Les Américains, avec pres de 3 millions d'entrées à nos frontières par année, demeurent la clientèle sur laquelle on doit concentrer les efforts de promotion du Québec comme destination touristique internationale. Les données de l'enquête sur le marché ané. ricain des woyages d'agrément constituent des outils de base pour la segmentation des marchếs. Quant à la clientẻle touristique internationale non-américaine, malgré sa croissance depuis 8-10 ans, elle ne constitue encore qu'un segment de marché relativement faible, pour lequel il faudra recueillir davantage d'informations avant de concevoir des stratégies marketing de façon concertée et efficace. $f$

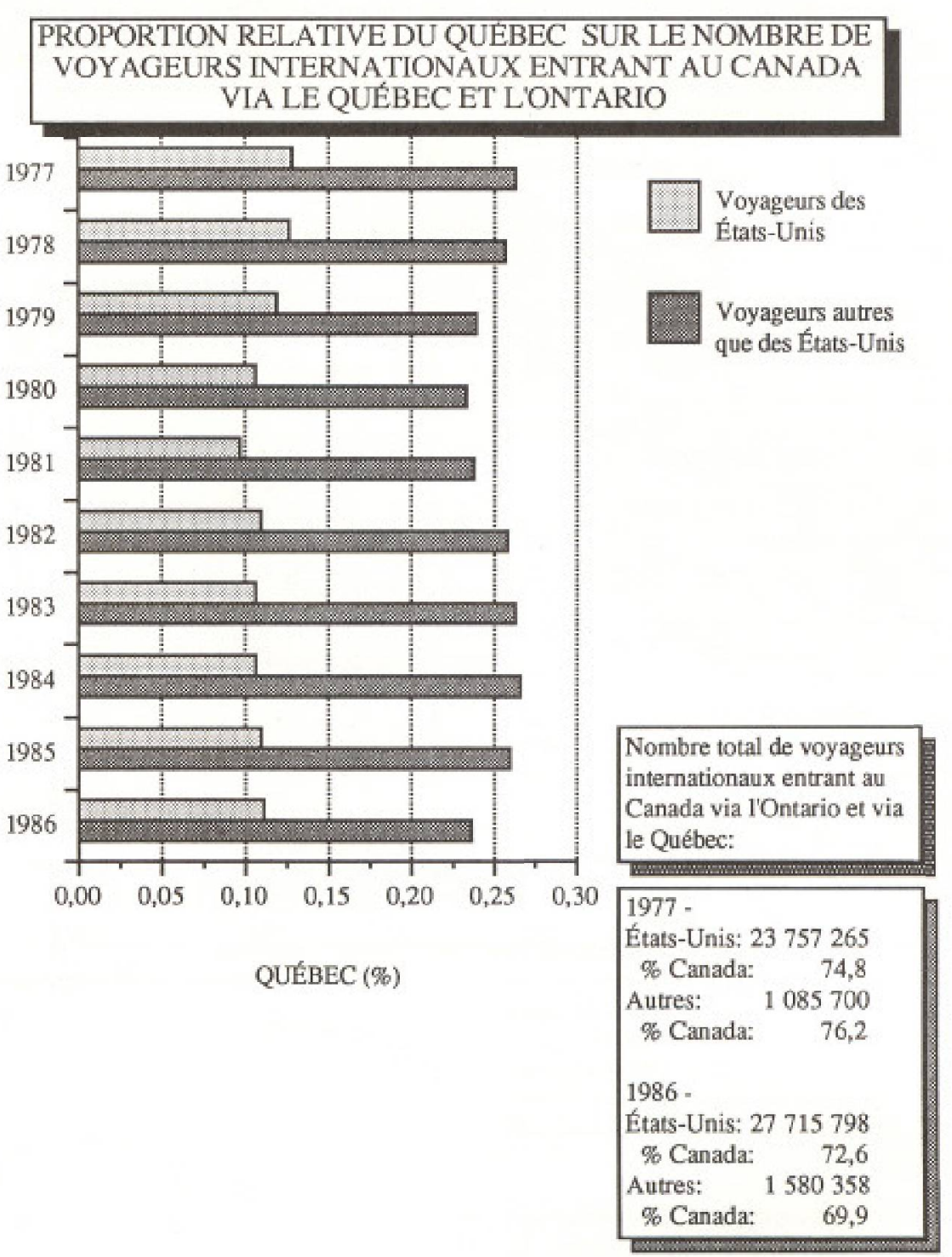

GRAPHIQUE 5 